Didáctica. Lengua y literatura

ISSN: 1130-0531

http://dx.doi.org/10.5209/DIDA.61964

\title{
Innovación e investigación en la elaboración de proyectos europeos plurilingües aplicados a la didáctica de las CC.SS, las lenguas y las literaturas
}

\author{
Ramón Tena Fernández ${ }^{1}$
}

Recibido: 5 de abril de 2016 / Aceptado: 18 de octubre de 2017

Resumen. La presente propuesta didáctica se define como un proyecto plurilingüe internacional, cuya finalidad es que escolares españoles por medio de su capacidad creativa den a conocer a través de cuentos digitales, su cultura y su lengua a estudiantes de cualquier otro país. Pues, de forma muy participativa e interdependiente a la par que nuestros alumnos ofrecen íntegramente su esencia cultural, los del otro país colaborador harán exactamente lo mismo. De modo, que al acabar la experiencia ambos colegios puedan editar de forma conjunta una obra literaria de carácter juvenil, con un gran potencial didáctico y puntera en su metodología de desarrollo, ofreciéndose mutuamente un novedoso aprendizaje de lenguas.

Palabras clave: ELE; internacional; LIJ; digital; cultural; lenguas.

\section{[en] Innovation and Research in the Development of Multilingual European Projects Applied to Teaching Social Science, Languages and Literatures}

\begin{abstract}
This didactic proposal is defined as an international multilingualism project whose aim is that Spanish pupils will make other pupils from different countries aware of their digital tales, language and culture throughout his creativity. Thus, in a very independent and collaborative way, our students will offer its cultural essence, and the partner country's students will do exactly the same. Therefore, at the end of the experience, both schools will edit a literary juvenile tale together with a great educational potential, also leader in its development methodology, thus offering each other innovative language learning.
\end{abstract}

Keywords: ELE; international; LIJ; digital; cultural; languages.

[fr] L'innovation et la recherche dans le développement de projets européens multilingues appliqués à l'enseignement des sciences sociales, les langues et les littératures

Résumé. Notre intention est d'aller vers un renouvellement complet dans l'enseignement lingüistique et culturel de la langue française en Espagne, et parralèlement proposer une autre alternative de l'enseignement ELE dans les centres français. Ce projet est le résultat de nombreuses années de préparation et fait découvir un nouveau cas de figure réelle de practique en classe, tout comme de nouveaux pro-

\footnotetext{
1 Dpto. de Didácticas de las CC.SS, las lenguas y las literaturas. Facultad de Formación del Profesorado. Universidad de Extremadura (España)

E-mail rtena@unex.es
} 
cédés, outils et indices de qualité. Tout cela sera à la portée de tout enseignant dès la diffusion de nos résultats. Le projet qu'il présente aujourd'hui, est un projet odacieux, moderne et pionnier. Il conjugue l'apprentissage du français et de l'espagnol avec la nouvelle technologie. Les élèves des deux pays de référence en seront les protagonistes et seront interactifs entre eux, tout cela malgré la chaîne des Pyrénées qui les séparent.

Mots-clés: ELE; international; LIJ; digital; la culture; la langue.

Sumario. 1. Introducción. 2. Justificación. 3. Propósitos e intencionalidades. 4. Plan de acción. 4.1. Planteamiento. 4.2. Secuenciación. 4.3. Visibilidad e impacto. 5. Bibliografía.

Cómo citar: Tena Fernández, R. (2018) Innovación e investigación en la elaboración de proyectos europeos plurilingües aplicados a la didáctica de las CC.SS, las lenguas y las literaturas, Didáctica. Lengua y literatura, 30, 221-230.

\section{Introducción}

El sistema educativo europeo no universitario ha asumido que vivimos en una sociedad en continuo cambio, por ello, la labor docente tiene que hacer frente cada día a nuevas demandas sociales y necesidades académicas. Así, el carácter heterogéneo del alumnado escolar, cada vez más definido por la pluralidad de etnias, razas y nacionalidades requiere de intervenciones concretas que den respuesta a este hecho (MECD, 2006). Esta compleja realidad exige una actualización permanente de las competencias profesionales docentes, que requerirá de una constante renovación de la formación universitaria original. Formación permanente que puede conseguirse si nos asomamos a las características pedagógicas que se emplean en los países de los que proviene su grupo de alumnos (Ruiz Garrido y Saorín Iborra, 2009).

De este modo, con la intención de impartir una enseñanza de calidad, global y plural, la investigación y la cooperación entre los centros educativos deben ir de la mano, más aún entre países vecinos como España y Francia, donde los aspectos culturales comunes permitirán resultados asombrosos (Cassany, 2005). Por este motivo, debemos incentivar programas de aulas abiertas en los que se favorezca el intercambio de experiencias y el descubrimiento de nuevas pautas pedagógicas, como el que mostramos a continuación. Entendemos que con su adaptación a los diversos sistemas educativos, los profesores tendrán la posibilidad de realizar proyectos internacionales con los que no solo mejoren su competencia comunicativa en otras lenguas, sino que también aprenderán nuevas estrategias didácticas para implementar en sus aulas (Madrid Manrique, 2014).

Tomando como punto de partida este hecho, para este trabajo en particular, nos proponemos ahondar en los convenios existentes entre España y Francia en materia educativa. Puesto que actualmente entre ambos países existen programas de estancias profesionales docentes e intercambios de auxiliares de conversación con los que se pretende incentivar un enriquecimiento mutuo en lo que respecta a filosofías de aprendizaje. Aprovechando esta infraestructura podremos establecer una enseñanza eficaz de ELE para los hablantes francófonos y una didáctica FLE efectiva para los hispanohablantes. A su vez, este proyecto pretende alcanzar estos dos objetivos por 
medio de una temática común, como es la Literatura Infantil y Juvenil, de modo que conecte el trabajo de la trama lingüística de ambas culturas.

La enseñanza del español como lengua extranjera, gracias a la encomiable labor de Instituciones como el Ministerio de Educación y el Instituto Cervantes, va cobrando un posicionamiento e interés cada día más destacado entre los alumnos de los países miembro de la Unión Europea y del mundo en general. No obstante, si bien los docentes hemos de estar capacitados y formados en este ámbito educativo, podemos aprovechar los numerosos planes de movilidad promovidos por diversas becas, con la intención de conocer en primera instancia los sistemas educativos del alumnado de ambos países, ya que es un hecho habitual el encuentro en colegios de ambos lados de los Pirineos de alumnos españoles en Francia y a la inversa (Suso López, 2001). A lo que debemos sumar la elevada tasa de escolares inmigrantes que demandan la reserva de algunas horas lectivas dedicadas exclusivamente a la enseñanza del español, de manera que se les facilite su integración social y académica dentro del centro educativo (Galindo Merino, 2012). Por consiguiente, ya que trabajamos con estos alumnos tan heterogéneos y con sus centros escolares de origen ¿Por qué no crear un proyecto que ayude a aumentar el prestigio de nuestro colegio y por ende el de su equipo docente?

Esa es nuestra intención, mostrar un ejemplo viable de proyecto intercentro, que cuente con procedimientos, herramientas e índices de calidad, como demandan las instituciones implicadas en este proceso de enseñanza-aprendizaje (Valverde Berrocoso, 2015). De esta manera, podemos conseguir que aquellos maestros vocacionales interesados en su implementación editen sus propias propuestas, potencien sus capacidades y desarrollen estas competencias profesionales. Así las cosas, con este trabajo queremos dar respuesta a algunas cuestiones que nos preocupan dentro de la enseñanza de ELE y en las secciones de plurilingüismo como las que enumeramos a continuación: ¿Cómo desarrollar la innovación? o ¿Qué actividades originales podemos realizar dentro del aula? Comencemos por el principio.

\section{Justificación}

¿Por qué desarrollar proyectos de esta naturaleza? La justificación de este tipo de propuestas didácticas las encontramos en el Informe del Consejo y la Comisión Europea de marzo de 2012 sobre la aplicación del marco estratégico en el campo de la educación y la formación. Aquí se reconoce la necesidad de movilidad del profesorado, puesto que es una línea de formación que contribuirá al crecimiento docente de los países miembros para la próxima década. Tomando como punto de partida esta necesidad, la Dirección General de Educación y Cultura de la Comisión Europea reconoce la urgencia de actualizar las competencias y cualificaciones del profesorado. Por tanto, se pretende impulsar investigaciones educativas que señalen metodologías, estrategias didácticas y valores pedagógicos de países vecinos como Francia o Portugal para que posteriormente, mediante colaboraciones mutuas, podamos instaurar en nuestras aulas los recursos e iniciativas más recomendables (Arnau, 1992).

De este modo, amparándonos en las demandas educativas de la Estrategia 2020 y en la Política Cultural de la Comisión Europea, encontramos la justificación de nuestra experiencia didáctica. Su pertinencia quiere proponer la práctica de meto- 
dologías renovadoras que impulsen la implicación del alumnado y contribuyan a suplir las necesidades formativas que a día de hoy tiene el sistema educativo actual (Gargiulo Hebe, 2015). Es por ello que entendemos que el potencial didáctico de la Literatura Infantil y Juvenil, como medio de enseñanza de las lenguas extranjeras, puede ser una línea de trabajo que ayude a encontrar puntos de unión entre las intenciones de mejora educativa que plantean las instituciones europeas y las deficiencias que el sistema muestra (Pascua Flebes, 2007).

A través de los resultados que arroje la puesta en marcha de actuaciones como la que queremos mostrar, los docentes serán conocedores del índice de impacto de esta metodología en la enseñanza de segundas lenguas, al tiempo que sus centros educativos tendrán evidencias que fomenten la autonomía de los escolares e incentiven el aprendizaje de los aspectos culturales más relevantes de España y Francia (lo cual podría ampliarse a otros países que tuvieran a bien involucrarse en esta forma de trabajo). Por otra parte, creemos que los resultados obtenidos serán útiles a nivel estatal, ya que puede mostrar un ejemplo real de aula que evidencie cómo, una vez finalizados los periodos de disfrutes de becas y estancias profesionales, se aumenta el prestigio docente y se posibilita la conformación de redes con las que crear grandes proyectos de reconocimiento europeo.

Por último, el uso continuado de las tres competencias que los escolares han de poner en práctica a lo largo de todo nuestro plan de acción es otro valor añadido que justifica la conveniencia de esta iniciativa. Según los últimos resultados de la OCDE, PISA y TALIS, las competencias lectora, tecnológica e idiomática, representan los pilares que hemos de reforzar si deseamos adaptarnos a las necesidades reales que demanda nuestra sociedad, hecho que, como veremos a continuación, se ha tenido en cuenta en cada una de las fases que plantea el proyecto (García Aretio, 2014).

\section{Propósitos e intencionalidades}

Los objetivos y fines que rigen la presente propuesta están enmarcados dentro de la iniciativa emblemática Juventud en Movimiento, ideada por la Comisión y el Consejo Europeo con la intencionalidad de impulsar la formación competitiva y de calidad tanto de profesores como de alumnos para el 2020. Por otra parte, la concreción de cada uno de estos objetivos ha sido inspirada en la metodología AICLE, ideada específicamente para el aprendizaje de lenguas extranjeras y con la que pretendemos trabajar simultáneamente contenidos culturales y sociales de los países francófonos, a la par que estos harán exactamente lo mismo con los españoles.

El desarrollo y ejecución de las pautas que desvelaremos en líneas sucesivas no solo han de velar por generar conocimiento y resultados de calidad en el campo de la didáctica de las lenguas y las literaturas, sino también por adquirir variedad de Recursos Educativos en Abierto (REA) que posteriormente puedan ser difundidos tanto en plataformas europeas como nacionales (sirva de ejemplo PROCOMUN). El propósito es que docentes con inquietudes similares no tengan por qué empezar de cero, al tener la posibilidad de poder editar y reutilizar los materiales depositados en estos bancos de recursos. De este modo se facilita tanto la mejora de los resultados de experiencias iniciales, como también la especialización de las herramientas ya utilizadas. 
Aunque a rasgos generales pensamos que es idónea la finalidad que plantea el proyecto, conviene concretar los objetivos de forma más precisa y detallada, de tal modo que podamos delimitar nuestro campo de acción. En este sentido, apoyándonos en un barómetro que nos ayude a graduar y priorizar nuestras intenciones, comenzaremos por referenciar como propósito principal el interés por crear, valorar y evaluar de forma consensuada entre centros educativos franceses y españoles una nueva propuesta metodológica de aprendizaje centrada en la enseñanza ELE y FLE (Jean-Pierre, 2011).

Este nuevo método de enseñanza presenta como eje principal la Literatura Infantil y Juvenil, núcleo del proyecto que se erige como fuente de conocimiento del léxico y la cultura en el aprendizaje de lenguas extranjeras y del que pretendemos conocer su potencial didáctico en la enseñanza de idiomas (Barcia Mendo y Soto Vázquez, 2010). En concreto, recurriremos a la narración denominada Digital Storytelling, con la que crearemos narraciones digitales tanto en francés como en español, utilizando la lengua española como vehicular con la intención de crear comunidades de aprendizaje conectadas en RED y así poner en práctica la parte oral que conlleva la didáctica de cualquier lengua (Regueiro Rodríguez, 2014).

Esta defensa por el aprendizaje conectado en tiempos de redes nos ayudará a potenciar el dominio de Flipped Classroom, la gran apuesta finlandesa para la mejora de la enseñanza que está invirtiendo los roles del aula. Es decir, los alumnos son los que se convierten en generadores del conocimiento, trabajan de forma autónoma los contenidos en su casa, los amplían en el aula y los comparten con sus compañeros mediante la edición de sus propios vídeos (Pérez Gómez, 2012). El hecho de convertir el ámbito doméstico en la primera toma de contacto con los nuevos contenidos conllevará una mayor implicación por parte de la familia, ya que serán ellos los que satisfagan las dudas iniciales de los escolares, factor que no implica ceder las tareas del profesorado a los padres, todo lo contrario. Se pretende que la familia sea consciente y participe del aprendizaje que va adquiriendo su propio hijo, ayudándole a detectar sus necesidades formativas e inquietudes temáticas, de modo que posteriormente se las puedan trasladar al docente, contribuyendo así a que la inversión del tiempo en cada materia sea más eficaz.

Además, al ser los escolares los generadores de los vídeos explicativos ayudarán a simplificar el proceso de enseñanza porque los conceptos son desgranados por medio de un diálogo establecido entre iguales, de alumno a alumno. Por tanto, el léxico será acorde a sus capacidades e intereses, potencial didáctico que se verá reforzado al crear los podcast en la plataforma digital del aula, donde cada niño puede plantear abiertamente sus dudas y el resto de compañeros, bajo la moderación y supervisión del tutor, puede presentar sus propuestas de soluciones, lo que favorecerá un aprendizaje colectivo (Martínez Rodrigo, 2008).

Tampoco debemos obviar que los alumnos del siglo XXI han crecido en un entorno digital, su grado de familiaridad con las nuevas tecnologías les predispone para un entorno educativo más motivador y cercano (MECD, 2010). Por ello, para la edición y maquetado de vídeos que requiere el empleo de la metodología Flipped classroom, nos vamos a apoyar en la disciplina Mobile Learning, donde tendrá cabida el empleo de aplicaciones relativas a la realidad aumentada. De este modo, no solo daremos vida a los personajes de nuestras narraciones digitales, sino que también favoreceremos la visibilidad e impacto de los productos creados entre los miembros de la comunidad educativa, que podrán ser conocedores en cualquier parte del mundo del material generado en el transcurso del proyecto. 
Como breve recapitulación, podemos establecer que la finalidad principal es trabajar la enseñanza FLE y ELE, por medio de un cuento bilingüe de creación propia entre centros educativos de sendos países. Además, proponemos desarrollar este gran reto con la incorporación de las tres metodologías defendidas por el Instituto Nacional de Tecnologías Educativas y Formación del Profesorado: Digital Storytelling para la narración oral, Flipped Classroom para la parte cinematográfica de las aventuras descritas y Mobile Learning para la edición de las aventuras en realidad aumentada. ¿Cómo integrar todos estos propósitos de manera ordenada y coherente?

Por medio de ABP (Aprendizaje Basado en Proyectos), metodología integradora del resto, fraccionaremos nuestro objetivo final en una serie de pequeñas metas, que exigirá tanto el compromiso de los centros implicados como la interdependencia positiva por parte de alumnos, profesores y familia (Escribano y Del Valle, 2008). De este modo, cada semana se planteará la resolución de un objetivo diferente relacionado con la creación de una parte esencial del cuento y que, por ende, conllevará la instauración de una nueva metodología, con este cambio evitaremos repetir ocupaciones, grupos de trabajo y metas a alcanzar.

\section{Plan de acción}

\subsection{Planteamiento}

En primer lugar, debemos tener en cuenta que si queremos fomentar una implicación real por parte de los alumnos tendremos que apostar tanto por sus puntos de interés, como por contenidos novedosos que capten su atención desde el primer segundo. Por ello, proponemos ligar temas que normalmente se descuidan en la enseñanza de lenguas extranjeras, pero que resultan atrayentes para los estudiantes. Además, conviene presentarlos bajo un formato innovador, que incentive la predisposición al trabajo, por tanto, nos apoyaremos en la secuencia de retos que dictamina la filosofía $\mathrm{ABP}$, de esta manera encadenaremos todas las actividades de forma coherente y significativa.

En lo que respecta a los centros de interés, proponemos la enseñanza de la lengua bajo el eje vertebrador de la Literatura Infantil y Juvenil (LIJ) porque aparte de resultar atractivo para todas las edades, nos va a permitir trabajar plenamente las cuatro destrezas competenciales de una lengua (Leer, Escribir, Escuchar y Hablar) (Soto Vázquez y Barcia Mendo, 2014). Y lo que es más importante, se presta a tratar los temas "descuidados" a los que aludíamos anteriormente, como pueden ser los aspectos culturales y sociales de los países de habla hispana para la enseñanza ELE o los europeos para las secciones bilingües (López Tamés, 1990).

Queremos que nuestros alumnos conozcan los elementos más emblemáticos de cada país. Es decir, sus fiestas, monumentos, gastronomía, museos, parques de atracciones y costumbres más representativas. En base a ellas ejercitarán las competencias claves oportunas para su correcto conocimiento y comprensión de la lengua en cuestión ¿Cómo trabajaremos todos estos temas? Pues, por medio de un cuento encadenado que incentive la libre creatividad de nuestros alumnos y que crearemos de forma conjunta entre nuestro país y el de referencia de los escolares extranjeros. Con la peculiaridad de que alternativamente cada mes dará continuidad al relato un 
centro de cada región o Comunidad Autónoma diferente y que se comprometerá a participar en este proyecto de forma activa y unánime.

¿Por qué esta heterogeneidad de centros? Porque la cultura de todo un país no se puede representar solo con las características de una determinada provincia y mucho menos su acento, y eso es precisamente lo que buscamos, que los alumnos comprendan y trabajen las variaciones que puede haber dentro de una misma lengua en función de la procedencia de sus hablantes. Al participar centros de diversos puntos geográficos la trama argumentativa se desarrollará en los ámbitos más significativos y característicos de cada uno de ellos, hecho que favorecerá una visión más global, plural y completa de la cultura y la lengua de los países involucrados.

Como eje vertebrador inventaremos un personaje común, el protagonista de nuestra historia, que ejercerá de hilo conductor en el argumento creado por cada región o localidad implicada y que además irá cambiando sus atuendos, costumbres y alimentación en función de sus nuevos destinos. El resto de personajes secundarios pueden ser animales característicos de cada región o mascotas emblemáticas de toda una generación, de modo que al acabar de editar la obra su lectura resulte amena y útil para impregnarse de todo un crisol de culturas.

¿Qué puntos fuertes presenta este proyecto? El trabajo de las cuatro destrezas esenciales en el aprendizaje de cualquier lengua, pero de forma integrada, con sentido y coherencia, conociendo de forma clara y específica la parte útil de cada competencia. Es decir, los alumnos van a comprender por sí mismos por qué y para qué aprender. Por otra parte, si nos centramos en el curriculum LOMCE, nos damos cuenta de que nuestro proyecto se encuentra avalado por los nuevos paradigmas que articula esta ley educativa, ya que entre las sugerencias acerca de cómo proceder con las competencias claves encontramos una perfecta definición de nuestra propuesta de actuación:

Para una adquisición eficaz de las competencias y su integración efectiva en el currículo, deberán diseñarse actividades de aprendizaje integradas que permitan al alumnado avanzar hacia los resultados de aprendizaje de más de una competencia al mismo tiempo.

\subsection{Secuenciación}

Toda esta literatura escrita resulta atractiva para justificar nuestras pautas didácticas pero ¿cómo las llevamos a cabo? ¿Cómo ordenamos las destrezas y competencias implicadas? Primeramente, motivaremos a nuestros alumnos para que ejecuten un impecable trabajo documental en el que recopilen los aspectos más reseñables de su cultura, de modo que tras una eficaz curación de contenidos puedan convertir sus señas de identidad en argumentos del cuento que van a editar. Seguidamente, para representar lo narrado y dinamizar las aventuras, los escolares serán los que ilustrarán mediante imágenes, collages y fotografías los monumentos, paisajes y ciudades que el protagonista vaya visitando, así como todo aquello que identifique el patrimonio arquitectónico, social y cultural de ese país.

Se debe destacar además, que el desarrollo de este proyecto resulta atractivo tanto para alumnos como para docentes, pues tiene un componente creativo que despierta interés e intriga. Normalmente los centros participantes tienden a encontrase "in- 
quietos" tras su aportación al libro, ya que desconocen por completo de qué forma y modo el colegio sucesor dará continuidad a las aventuras que ellos han iniciado previamente.

¿Dónde radica la novedad de este proyecto? Aparte de trabajar cooperativamente de forma internacional e interdependiente para alcanzar una meta común, nos encontramos con que los alumnos son los generadores de su propio aprendizaje, contenidos y recursos. Porque los capítulos editados en España por las diversas Comunidades Autónomas sirven para la enseñanza del ELE a centros extranjeros y viceversa con los colegios europeos (Lorenzo Bergillos, 2011).

Además, a este proyecto colaborativo lo sustenta también el guiño hacia la ferviente apuesta de la LOMCE por potenciar sobremanera la competencia lingüistica y la tecnológica. De modo que, con la intencionalidad de otorgarle el protagonismo que se merecen estas competencias en el aprendizaje a lo largo de la vida de nuestros estudiantes, una vez concluida la fase de redacción y edición del libro, se estima necesaria la elaboración de un vídeo documental a modo de película.

Su conveniencia radica en que trabajamos simultáneamente las dos competencias prioritarias, ya que serán los escolares los que den voz propia a los personajes del cuento y por ende manejen herramientas y recursos digitales que aunque de forma intuitiva les harán actualizarse y ejercitarse tecnológicamente para el futuro.

Tampoco podemos olvidar que estamos trabajando con nativos digitales y esta opción se presenta atractiva y motivadora para todos sus participantes que se encuentran cualificados en el uso de las TIC (Vera Pérez, 2002). Sin perder de vista la finalidad de esta propuesta: potenciar la enseñanza ELE y la familiarización con el plurilingüismo, hecho que cumplimos si tenemos en cuenta que los audios del vídeo son realizados por hablantes originarios de cada país participante. Por tanto, trabajamos con nuestros alumnos habilidades de escucha y de comunicación de la mejor forma posible familiarizando su oído y pronunciación de manera específica y profesional desde edades muy tempranas.

4.3. Visibilidad e impacto

Por otra parte, hemos de resaltar que con la creación del vídeo potenciamos la difusión y repercusión del material creado, ya que una vez concluido puede ser expuesto en plataformas educativas como PROCOMUN. De este modo, finalizado nuestro proyecto podemos seguir buscando nuevos centros a los que les interese la temática y la metodología expuesta, con ello inmortalizaremos a los personajes que continuarán viviendo nuevas aventuras en países diferentes, y por tanto, ampliando el potencial plurilingüe y la riqueza cultural de nuestros alumnos.

Toda la literatura didáctica de páginas anteriores con la que hemos descrito las fases que articulan este plan de acción puede inducir a que muchos docentes piensen que un proyecto de estas dimensiones es poco factible y muy complejo. No obstante, aunque suene fantasioso se podría decir que con vocación, entusiasmo y compromiso pedagógico, el desarrollo correcto de proyectos de esta naturaleza es francamente fácil. ¿Por qué?

Porque los profesionales de la enseñanza cuentan con Etwinning una plataforma gratuita y segura que permite a los docentes compartir ideas y desarrollar proyectos colaborativos en Europa. La utilización de esta plataforma es conveniente si tenemos en cuenta que se encuentra traducida a veintiséis idiomas y alberga más de doscientos mil miembros relacionados con el ámbito de la educación, hecho positivo no solo para comenzar el proyecto sino para darle continuidad ¿Cómo? 
De forma muy sencilla, dado que una vez creado el primer volumen de nuestra serie de cuentos y editado el vídeo, podremos darlo a conocer tanto en la web, como en los encuentros internacionales Etwinning. Porque, este espacio contribuye a que los compañeros europeos interesados en nuestro resultado inicial, carezcan de dificultades para contactar con los precursores y unirse al proyecto para su ampliación y continuación.

Además, aparte del factor apasionante y entretenido que tiene el hecho de embarcarse en este tipo de aventuras, existe un motivo añadido que incentiva aún más su realización, pues tal y como vaticinamos al inicio de este artículo, también podemos aumentar nuestro prestigio profesional y el de nuestro colegio. Cada año, la mencionada comunidad de centros escolares se encarga de seleccionar los proyectos de mejor calidad, para otorgarle premios y reconocimientos de distinción europea, incrementando de este modo el empoderamiento social y el prestigio académico de la entidad galardonada. Lo mismo sucede a nivel nacional, pues, el MECD por medio de convocatorias específicas como PROMECE facilita y promueve la asociación de centros educativos de diferentes CC.AA, para que puedan desarrollar colectivamente un mismo proyecto común.

Por tanto, trabajar mediante ABP de forma internacional y apostando fervientemente por las competencias claves relacionadas con la lectura y las nuevas tecnologías (las más defendidas por LOMCE) sólo es cuestión de interés y ganas. La enseñanza ELE y las secciones bilingües merecen mucho más que desgastadas audiciones de casete sobre temas banales o rutinarias copias y dictados. Tenemos la formación necesaria, respaldo institucional y los medios requeridos para su ejecución, sólo necesitamos conocer las preferencias temáticas de nuestros alumnos y apostar por la innovación y la creatividad.

\section{Bibliografía}

Arnau, Joaquín (1992): La educación bilingüe, Barcelona, ICE / Universitat de Barcelona / Horsori.

Barcia Mendo, Enrique y José Soto Vázquez (2010): Glosario de literatura infantil y juvenil, Mérida, Servicio de Publicaciones de la Junta de Extremadura.

Cassany, Daniel (2005): Expresión escrita en L2/ELE, Madrid, Arco-Libros.

Courtillon, Janine (2003): Élaborer un cours de FLE, París, Hachette.

Escribano, Alicia y Ángela del Valle (2008): El aprendizaje basado en problemas (ABP): una propuesta metodológica en Educación Superior, Madrid, Narcea.

Galindo Merino, María del Mar (2012): La lengua materna en el aula de ELE, Madrid, Asociación para la Enseñanza del Español como Lengua Extranjera.

García Aretio, Lorenzo (2014): Bases, mediaciones y futuro de la educación a distancia en la sociedad digital, Madrid, Síntesis.

Gargiulo Hebe, Claudia (2015): Tecnología y metodología en la clase de ELE, Buenos Aires, Tinta fresca.

Jean-Pierre, Robert (2011): Faire classe en FLE: une approche actionnelle et pragmatique, Paris, Hachette.

López Tamés, Román (1990): Introducción a la literatura infantil, Murcia, Publicaciones Universidad de Murcia. 
Lorenzo Bergillos, Francisco José (2011): Educación bilingüe: integración de contenidos y segundas lenguas, Madrid, Síntesis.

Madrid Manrique, Marta (2014): La formación inicial del profesorado para la educación bilingüe, Granada, Editorial Universidad de Granada.

Martínez Rodrigo, Estrella (2008): Interactividad digital: nuevas estrategias en educación y comunicación, Madrid, EOS.

Ministerio de Educación y Ciencia, Subdirección General de Información (2006): Aprendizaje integrado de contenidos y lenguas (AICLE) en el contexto escolar europeo, Madrid, Servicio de Publicaciones MECD.

Ministerio de Educación, Secretaría General Técnica (2010): Nuevas formas de lectura en la era digital, Madrid, Publicaciones MECD.

Pascua Flebes, Isabel (2007): Literatura infantil para una educación intercultural: traducción y didáctica, Las Palmas de Gran Canaria, Servicio de Publicaciones Universidad de Las Palmas de Gran Canaria.

Pérez Gómez, Ángel I. (2012): Educarse en la era digital: la escuela educativa, Madrid, Morata.

Regueiro Rodríguez, María Luisa (2014): La programación didáctica ELE: pautas para el diseño de la programación de un curso ELE, Madrid, Arco-Libros.

Ruiz Garrido, Miguel y Ana María Saorín Iborra (2009): Hacia una educación plurilingüe: experiencias docentes AICLE, Castellón de la Plana, Universitat Jaume I.

Soto Vázquez, José y Enrique Barcia Mendo (2014): III Jornadas de Literatura Infantil y Juvenil en Extremadura, Cáceres, Diputación Provincial de Cáceres.

Suso López, Javier (2001): Discours, enonciation et enseignement-apprentissage du FLE, Granada, Método.

Valverde Berrocoso, Jesús (2015): El proyecto de educación digital en un centro educativo: guía para su elaboración y desarrollo, Madrid, Síntesis.

Vera Pérez, Carmen (2002): L'Internet en classe de FLE, Madrid, Longman / Pearson Educación. 